\title{
Negative Liquid Crystal Cell with Parallel Pattemed Electrodes for High Transmittance and Fast Switching
}

\author{
Joon Heo, Tae-Hoon Choi, Jae-Won Huh, and Tae-Hoon Yoon* \\ Department of Electronics Engineering, Pusan National University, Busan 609-735, Korea
}

(Received April 22, 2015 : revised May 26, 2015 : accepted May 29, 2015)

\begin{abstract}
We propose a negative liquid crystal (n-LC) cell with parallel patterned electrodes for high transmittance and fast response. The proposed cell has higher transmittance and shorter response time than a conventional fringe-field switching cell using n-LCs, because the proposed cell does not have transmittance dips and does have a thinner LC layer.
\end{abstract}

Keywords : Fast switching, High transmittance, Wide viewing angle, Liquid crystal, In-plane switching mode OCIS codes : (160.3710) Liquid crystals; (120.0120) Instrumentation, measurement, and metrology; (120.2040) Displays

\section{INTRODUCTION}

Liquid crystal displays (LCDs) are used widely in everyday life because of their various advantages such as light weight, low driving voltage, and low power consumption. Various efforts have been made to improve the quality of LCDs-for example, high transmittance, high resolution, high contrast, and wide viewing angle characteristics. Several liquid crystal (LC) modes, such as twisted nematic, vertical alignment, and in-plane switching (IPS), have been competing against one another in the LCD market [1-4].

Among the various LCD modes, the IPS mode is one of the mainstream technologies developed to achieve a wide viewing angle [4-9]. The IPS mode exhibits wide viewing angle characteristics because the LCs are rotated in a plane parallel to the glass substrates. However, the IPS mode suffers from low transmittance above the patterned electrodes.

To enhance the transmittance of the IPS mode, the fringefield switching (FFS) mode has been proposed [10-13]. The FFS mode exhibits higher transmittance than the IPS mode. Moreover, an FFS cell using LCs with negative dielectric anisotropy (n-LCs) exhibits higher transmittance than one using LCs with positive dielectric anisotropy (p-LCs). In addition, $\mathrm{n}-\mathrm{LC}$ can be driven at a low frame rate, because image flickering due to the flexoelectric effect is smaller in n-LCs than in p-LCs [14]. However, the transmittance above and between the patterned electrodes in an FFS cell using n-LCs still needs further improvement. Moreover, the response time of n-LCs is longer than that of $\mathrm{p}$-LCs because of the higher viscosity of the former. Several methods have been proposed to improve the transmittance and response time of the FFS and IPS modes [15-17]. However, these methods suffer from disadvantages such as complicated operation and high operating voltage.

In this paper, we propose an n-LC cell with parallel patterned electrodes for high transmittance and fast response. The proposed cell has a higher transmittance and a shorter response time than a conventional FFS cell using n-LCs, because the proposed cell does not have transmittance dips and has a thinner LC layer. It maintains the wide viewing angle characteristics of the conventional FFS mode using n-LCs.

\section{OPERATING PRINCIPLE}

To improve the electro-optical characteristics of a homogeneously aligned n-LC cell, we employed a double-sided patterned electrode structure [17-21], as shown in Fig. 1. The top and bottom patterned electrodes were parallel to each other, but their positions were shifted away from each other.

Figure 2 illustrates schematically the operating principle of the proposed cell. The operating principle of the proposed

\footnotetext{
*Corresponding author: thyoon@pusan.ac.kr

Color versions of one or more of the figures in this paper are available online.
} 


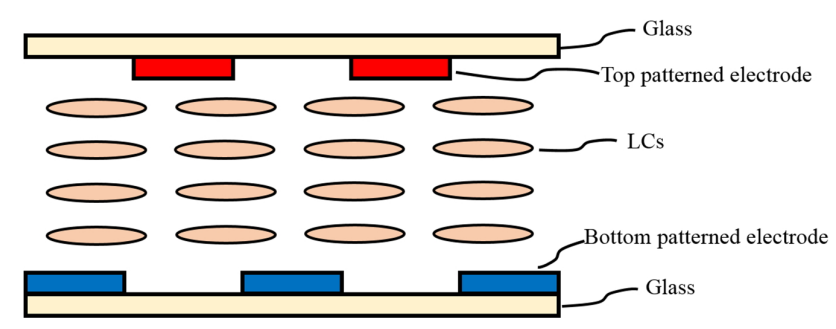

FIG. 1. Structure of the proposed cell.
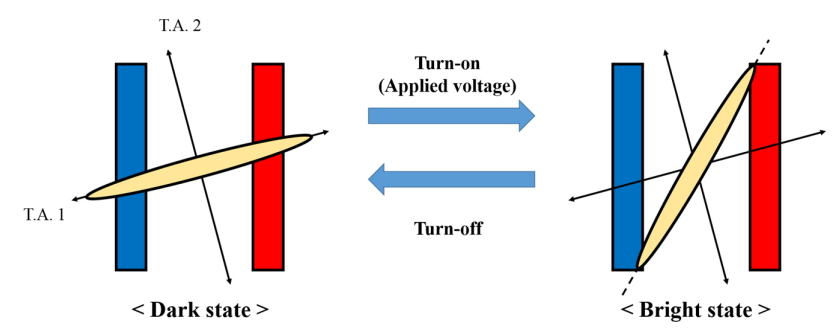

FIG. 2. Operating principle of the proposed cell.

cell is similar to that of the conventional FFS mode using n-LCs. In the initial state, the LCs are homogeneously aligned along one of the transmission axes of the crossed polarizers so that the cell is in the dark state. By applying different voltages to the top and bottom patterned electrodes, an oblique electric field is built into the LC layer. The LCs are rotated counterclockwise by the applied electric field, so that the proposed cell shows the bright state.

\section{SIMULATION CONDITIONS}

To confirm the electro-optical characteristics of the proposed cells, we used the commercial software TechWiz LCD 2D (Sanayi System Company, Ltd., Korea). The parameters used for numerical calculations were as follows. The width of the patterned electrodes and the gap between them were $2.5 \mu \mathrm{m}$ and $3 \mu \mathrm{m}$, respectively. The LC material parameters used in simulations were: $K_{11}=14.7 \mathrm{pN}, K_{22}=7.4 \mathrm{pN}$, $K_{33}=15.9 \mathrm{pN}, \Delta n=0.15$ (at a wavelength of $550 \mathrm{~nm}$ ), $\Delta$ $\varepsilon=-3.9$, and $\gamma_{1}=110 \mathrm{mPa}$ s. The n-LCs were homogeneously aligned and the pre-tilt angle was $0^{\circ}$. The rubbing direction was set to $80^{\circ}$ with respect to the patterned electrodes. The thickness of the LC layer was chosen for maximum transmittance at each condition. In addition, we studied the operating characteristics of the LC cell as we varied parameters such as the birefringence of the n-LCs, the width of the top patterned electrodes, and the gap between top patterned electrodes.

\section{RESULTS AND DISCUSSION}

We compared the transmittance of the proposed cell to that of the conventional FFS mode using n-LCs. The thicknesses of the LC layer for maximum transmittance in the proposed cell and the conventional FFS mode were 2.3 $\mu \mathrm{m}$ and $2.45 \mu \mathrm{m}$ respectively. The maximum transmittances of the proposed cell and the conventional FFS mode were $44.0 \%$ and $41.7 \%$ respectively. Figure 3 shows the transmittance distribution, potential profile, and LC director distribution of the conventional FFS mode and the proposed cell. As shown in Fig. 3, the transmittance distribution of the conventional FFS mode shows dips above and between the patterned electrodes; conversely, the transmittance of the proposed cell is nearly uniform. For this reason, the proposed cell demonstrated higher transmittance than the conventional FFS mode. In addition, the thickness of the LC layer for maximum transmittance of the proposed cell was smaller than that of the conventional FFS mode using n-LCs. In the conventional FFS mode, an electric field is applied between the patterned and common electrodes on the bottom substrate, so that the LC molecules far from the bottom substrate are not rotated. In the proposed cell,

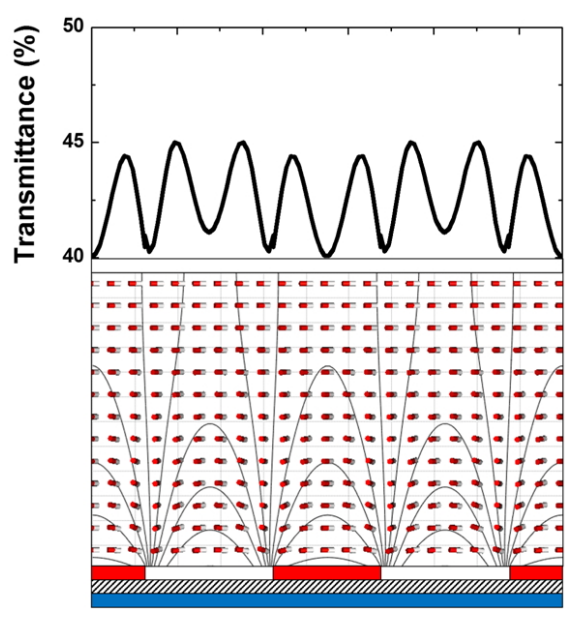

(a)

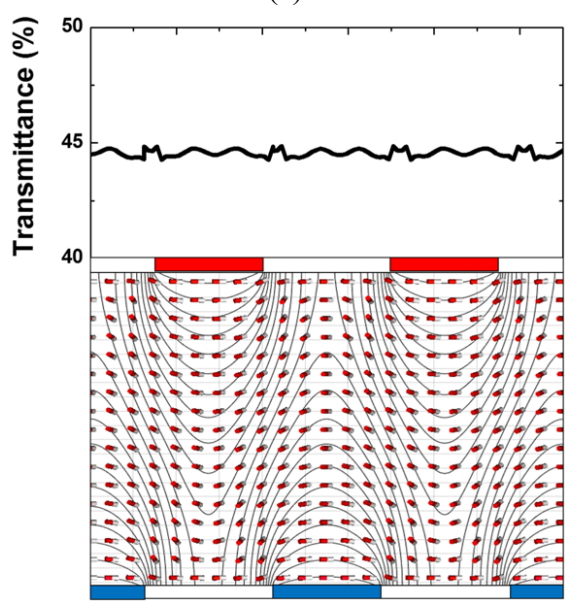

(b)

FIG. 3. Transmittance distribution, potential profile, and LC director distribution of (a) the conventional FFS mode and (b) the proposed cell. 
however, an electric field is applied between the top and bottom patterned electrodes, so that LC molecules everywhere can be rotated.

We compared the dependence of the transmittance on the gap between patterned electrodes. The birefringence of the n-LCs and the width of the patterned electrodes were 0.15 and $2.5 \mu \mathrm{m}$ respectively. As shown in Fig. 4(a), the proposed cell shows higher transmittance than the conventional FFS mode under all conditions. In addition, as the gap between patterned electrodes increased, the operating voltage of the proposed cell decreased, as shown in Fig. 4(b).

We compared the dependence of the transmittance on the birefringence of $n-L C s$. The thickness of the LC layer was chosen for maximum transmittance at each condition. The width of the patterned electrodes and the gap between them were $2.5 \mu \mathrm{m}$ and $3 \mu \mathrm{m}$ respectively. We assumed that all n-LCs have the same physical parameters except for the birefringence. As shown in Fig. 5, as the birefringence of the $\mathrm{n}$-LCs increased the transmittance of the conventional

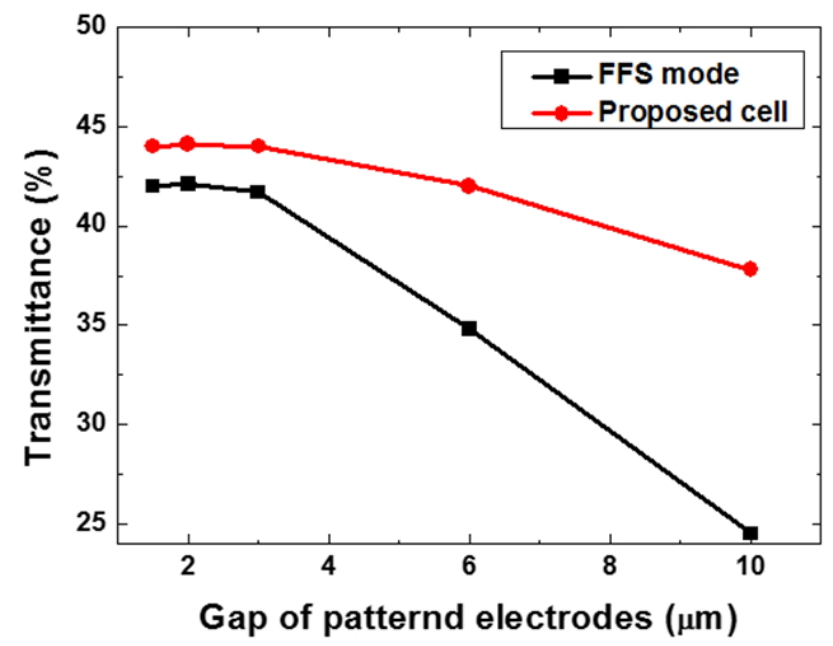

(a)

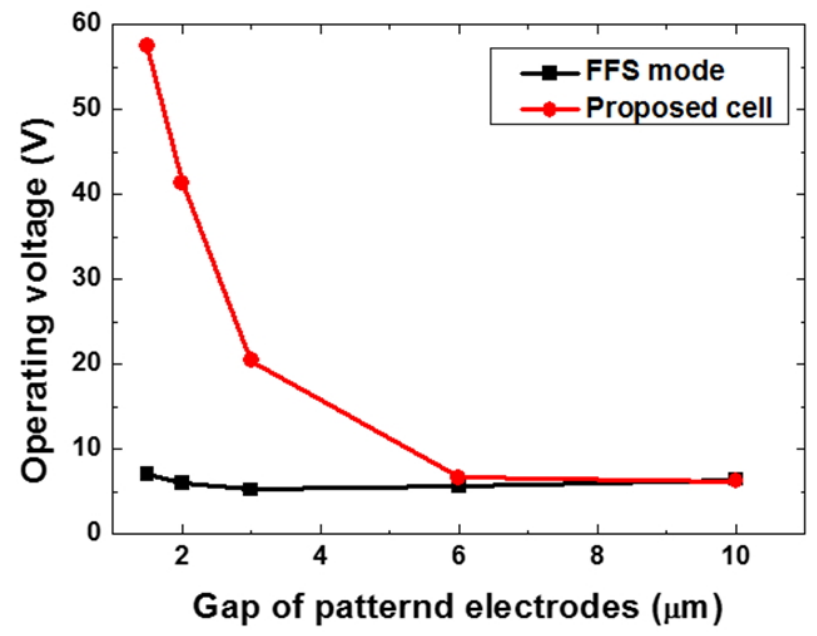

(b)

FIG. 4. Dependence of (a) the transmittance and (b) the operating voltage on the gap between patterned electrodes.
FFS mode decreased, because the LC molecules above and between the patterned electrodes were not rotated for small cell gaps. However, the transmittance of the proposed cell was nearly the same for every condition.

We compared the dependence of the electro-optical properties of the proposed cell on the width of the top patterned electrodes. The width of the bottom patterned electrodes and the gap between them were fixed at $2.5 \mu \mathrm{m}$ and $3 \mu \mathrm{m}$ respectively. The transmittance was maximized when the width of the top patterned electrodes was $3 \mu \mathrm{m}$, as shown in Fig. 6. As the width of the top patterned electrodes increased, the operating voltage increased, too.

We compared the response time of the proposed cell to that of the conventional FFS mode, as shown in Fig. 7. The response time of the proposed cell was shorter than that of the conventional FFS mode. The turn-on times of the proposed cell and the conventional FFS mode were the same at $8.2 \mathrm{~ms}$. The turn-off times of the proposed cell and the conventional FFS mode were $11 \mathrm{~ms}$ and $13.2 \mathrm{~ms}$

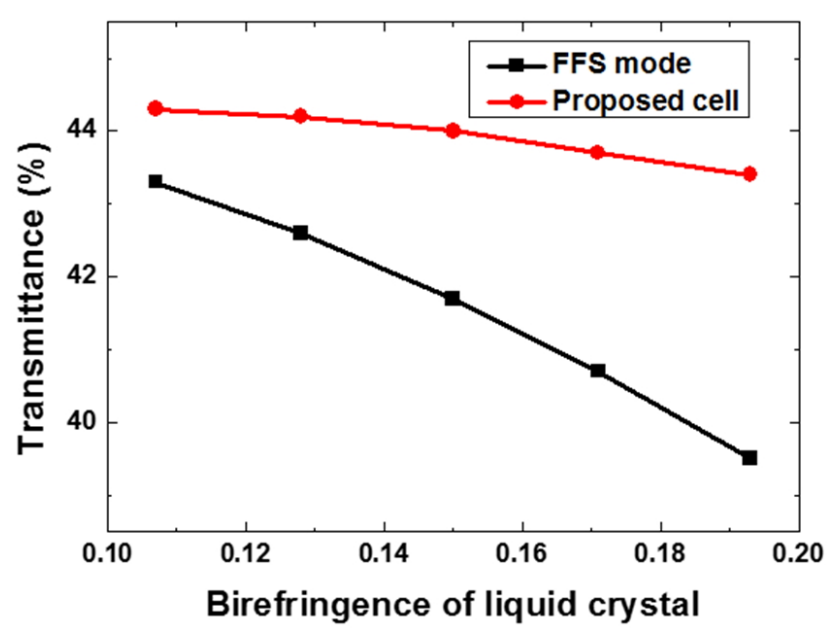

FIG. 5. Dependence of the transmittance on the birefringence of the LCs.

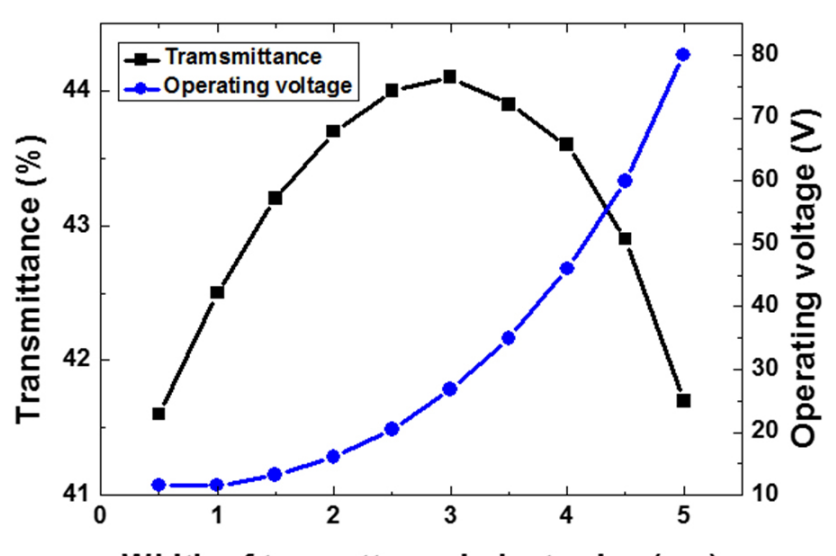

Width of top patterned electrodes $(\mu \mathrm{m})$

FIG. 6. Dependence of the transmittance and the operating voltage on electrode width in the proposed cell. 


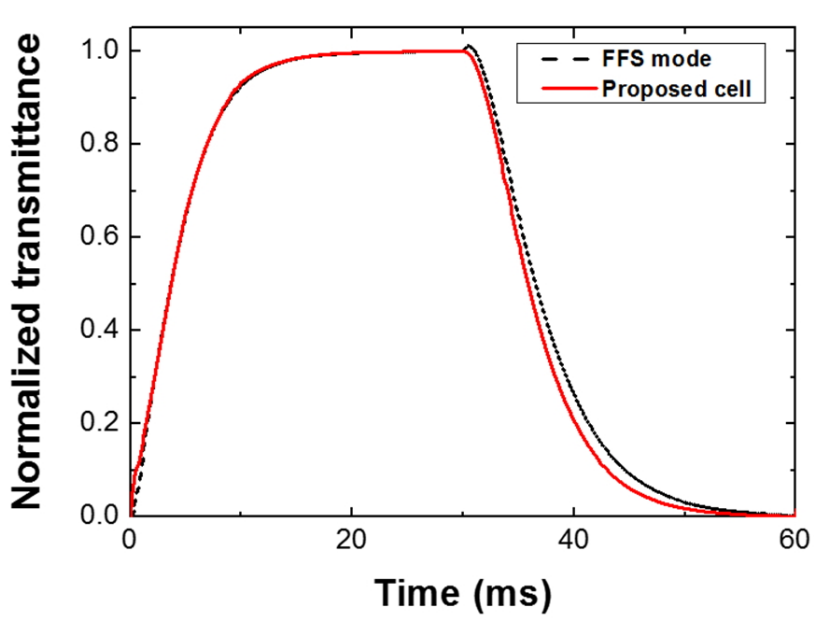

FIG. 7. Response time of the conventional FFS mode and the proposed cell.

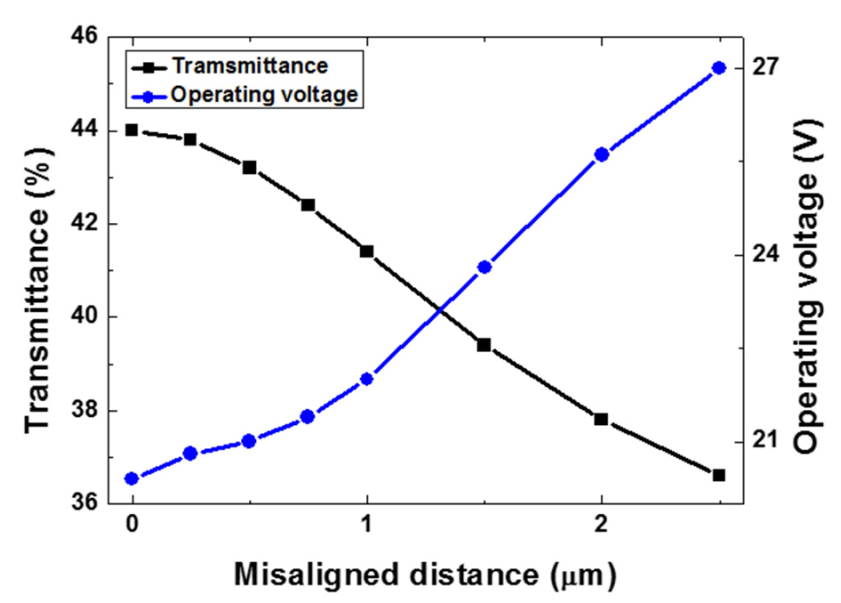

FIG. 8. Dependence of the transmittance and the operating voltage on misalignment in the proposed cell.

respectively. In the conventional FFS mode, LC molecules far from the bottom substrate were not rotated, because the electric field far from the bottom substrate was weak; conversely, in the proposed cell an electric field was applied between the top and bottom patterned electrodes, so that the LC molecules everywhere were rotated. Therefore, the proposed cell showed maximum transmittance at a smaller thickness of the LC layer than did the conventional FFS mode. When the proposed cell and the conventional FFS mode have the same cell gap of $2.3 \mu \mathrm{m}$, the turn-off times of the proposed cell and the conventional FFS mode were $11 \mathrm{~ms}$ and $11.6 \mathrm{~ms}$ respectively. Consequently, we can conclude that the difference in turn-off times between the two modes comes mainly from the cell-gap difference.

However, accurate alignment of the top and bottom patterned electrodes may be difficult in actual manufacturing processes. As shown in Fig. 8, the transmittance decreases and the operating voltage increases as the misalignment between
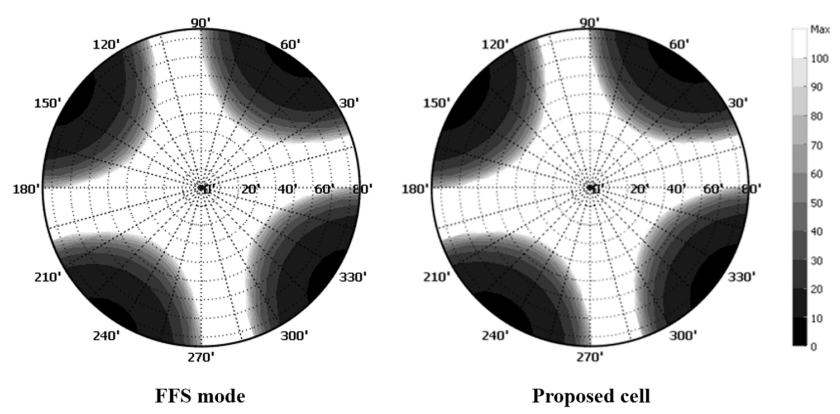

FIG. 9. Iso-contrast distribution of the conventional FFS mode and the proposed cell.

the top and bottom patterned electrodes increases. When the misalignment is $1 \mu \mathrm{m}$, the transmittance of the proposed cell is $41.4 \%, 5.9 \%$ lower than that of the aligned cell. Therefore, precise alignment is required for high performance of the proposed cell.

We calculated the iso-contrast distribution for the proposed cell and the conventional FFS mode. As shown in Fig. 9, the proposed cell exhibited wide viewing angle characteristics similar to those of the conventional FFS mode, because of the in-plane rotation of LC molecules.

\section{CONCLUSION}

In summary, we proposed an n-LC cell with parallel patterned electrodes for high transmittance and fast response. The proposed cell has shown higher transmittance than the conventional FFS mode using n-LCs, because the proposed cell does not have transmittance dips. In addition, the proposed cell has shown a shorter response time than the conventional FFS mode using n-LCs, because of smaller optimum thickness of the LC layer. In addition, the proposed cell exhibits wide viewing angle characteristics similar to those of the conventional FFS mode using n-LCs.

\section{ACKNOWLEDGMENT}

This work was supported by the National Research Foundation of Korea (NRF) grant funded by the Korean government (MSIP) (No. 2015021717).

\section{REFERENCES}

1. M. F. Schiekel and K. Fahrenschon, "Deformation of nematic liquid crystals with vertical orientation in electrical fields," Appl. Phys. Lett. 19, 391-393 (1971).

2. C. Z. van Doorn, "Dynamic behavior of twisted nematic liquid-crystal layers in switched fields," J. Appl. Phys. 46, 3738-3745 (1975).

3. M. Ohe and K. Kondo, "Electro-optical characteristics and 
switching behavior of the in-plane switching mode," Appl. Phys. Lett. 67, 3895-3897 (1995).

4. J.-W. Kim, T.-H. Choi, and T.-H. Yoon, "Design of an electrode structure for the elimination of the off-axis gamma shift in a multi-domain vertical alignment liquid crystal cell," J. Inf. Disp. 15, 19-23 (2014).

5. M. Ohe and K. Kondo, "Response mechanism of nematic liquid crystals using the in-plane switching mode," Appl. Phys. Lett. 69, 623-625 (1996).

6. M. Ohe, M. Yoneya, and K. Kondo, "Switching of negative and positive dielectro-anisotropic liquid crystals by in-plane electric fields," J. Appl. Phys. 82, 528-535 (1997).

7. S.-W. Oh, B. W. Park, J.-H. Lee, and T.-H. Yoon, "Achromatic optical compensation using dispersion of uniaxial films for elimination of off-axis light leakage in a liquid crystal cell," Appl. Opt. 52, 7785-7790 (2013).

8. S.-W. Oh and T.-H. Yoon, "Elimination of the light leakage over the entire viewing cone in a homogeneously-aligned liquid crystal cell," Opt. Express 22, 5808-5817 (2014).

9. S.-W. Oh, J.-H. Park, and T.-H. Yoon, "Near-zero pretilt alignment of liquid crystals using polyimide films doped with UV-curable polymer," Opt. Express 23, 1044-1051 (2015).

10. S. H. Lee, S. L. Lee, and H. Y. Kim, "Electro-optic characteristics and switching principle of a nematic liquid crystal cell controlled by fringe field switching," Appl. Phys. Lett. 73, 2881-2883 (1998).

11. Y. M. Jeon, I. S. Song, S. H. Lee, H. Y. Kim, S. Y. Kim, and Y. J. Lim, "Optimized electrode design to improve transmittance in the fringe-field switching (FFS) liquid crystal cell," SID Dig. Tech. Pap. 36, 328-331 (2005).

12. Y. Chen, Z. Luo, F. Peng, and S.-T. Wu, "Fringe-field switching with a negative dielectric anisotropy liquid crystal," J. Disp. Technol. 9, 74-77 (2013).

13. D. H. Kim, Y. J. Lim, D. E. Kim, H. Ren, S. H. Ahn, and S. H. Lee, "Past, present, and future of fringe-field switchingliquid crystal display," J. Inf. Disp. 15, 99-106 (2014).

14. L. M. Blinov and V. G. Chigrinov, Electrooptic Effects in Liquid Crystal Materials (Springer, New York, USA, 1994).

15. Z. Ge, X. Zhu, T. X. Wu, and S.-T. Wu. "High transmittance in-plane switching liquid crystal displays," J. Disp. Technol. 2, 114-120 (2006).

16. D. H. Song, K.-H. Kim, and T.-H. Yoon, "High-transmittance in-plane switching liquid crystal display device driven by three-level voltages," J. Soc. Inf. Disp. 21, 29-33 (2013).

17. T.-H. Choi, J.-W. Kim, and T.-H. Yoon, "Fast in-plane switching of negative liquid crystals using crossed patterned electrodes,” Jpn. J. Appl. Phys. 53, 081701 (2014).

18. M. Jiao, Z. Ge, S.-T. Wu, and W.-K. Choi, "Submillisecond response nematic liquid crystal modulators using dual fringe field switching in a vertically aligned cell," Appl. Phys. Lett. 92, 111101 (2008).

19. H.-C. Jau, P.-H. Liao, H.-W. Li, H.-K. Hsu, C.-H. Chen, C.-C. Wang, and T.-H. Lin, "Improvement of electro-optical properties of PSBP LCD using a double-sided IPS electrode," J. Soc. Inf. Disp. 20, 351-353 (2012).

20. C.-C. Li, H.-Y. Tseng, T.-W. Pai, Y.-C. Wu, W.-H. Hsu, H.-C. Jau, C.-W. Chen, and T.-H. Lin, "Bistable cholesteric liquid crystal light shutter with multielectrode driving," Appl. Opt. 53, 33-37 (2014).

21. J. Heo, J.-W. Huh, and T.-H. Yoon, "Fast-switching initiallytransparent liquid crystal light shutter with crossed patterned electrodes," AIP Adv. 5, 047118 (2015). 